\title{
Intravenous ibandronate in men with osteoporosis: An open pilot study over 2 years
}

\author{
O. Lamy, L. Sandini , I. Pache, S. Fatio, J. Burnand and P. Burckhardt \\ Division of Osteoporosis, Department of Internal Medicine, University Hospital, Lausanne, Switzerland
}

\begin{abstract}
In the treatment of osteoporosis, the tolerance of oral bisphosphonates is often low. The high potency of ibandronate allows iv bolus injections that can be repeated every 2 to 3 months. However, the best dose and time interval of the treatment with iv ibandronate is still debated. Efficacy of 2-mg ibandronate injected every 3 months was tested in men with osteoporosis over $2 \mathrm{yr}$, in a prospective, open study. Fourteen men with primary osteoporosis, mean age $57 \pm 12$ yr (range: $40-73$ ), received $2-\mathrm{mg}$ ibandronate iv every 3 months over 2 yr. All got $1 \mathrm{~g} /$ day calcium and $880 \mathrm{Ul} /$ day vitamin $D$ for 2 yr. Bone mineral density (BMD) increased after 2 yr by $6.7 \pm 1.5 \%$ (mean change \pm SEM) at lumbar spine $(p<0.001)$, by $3.2 \pm 08 \%$ at trochanter
\end{abstract}

\section{INTRODUCTION}

Osteoporosis is less common in men than in women, but approximately 25 to $30 \%$ of all hip fractures occur in men, and many men have vertebral deformities $(1,2)$. The causes of osteoporosis in men include excess of glucocorticoids, hypogonadism, and a variety of other systemic conditions, medications and lifestyle factors; in about $50 \%$ there is no obvious cause. Only few therapies have been evaluated in men. Testosterone therapy has limited efficacy in men with hypogonadism (3). Alendronate significantly increased spine and hip bone mineral density (BMD), and decreased the incidence of vertebral fractures (4). However, bisphosphonates cause gastrointestinal side effects in many patients (5), and must be taken under strict conditions, which affects compliance. These limitations led to the in-

Key-words: Bisphosphonate, bone mineral density, ibandronate, osteoporosis in men.

Correspondence: Olivier Lamy, MD, Department of Internal Medicine, CHUV, BH10 CH-1011 Lausanne, Switzerland.

E-mail: olivier.lamy@chuv.hospvd.ch

Accepted March 14, 2003. $(p<0.001)$ and by $1.4 \pm 1.1 \%$ at femoral neck (ns). Serum $\beta$-crosslaps and osteocalcin decreased significantly by $30-45$ and $30 \%$, respectively, during the $2 \mathrm{yr}$ of treatment. Serum calcium increased from the lower to the middle tertile of the normal range during the $2 \mathrm{yr}$ of the study. The observed decrease of bone remodelling and the increase of BMD are of the same magnitude as those described with oral bisphosphonates. The increase of plasma calcium confirms the positive effect of the supplementation with calcium and vitamin $D$. These results suggest that 3 months are a good interval between two doses of iv ibandronate, when $2 \mathrm{mg}$ are given.

(J. Endocrinol. Invest. 26: 728-732, 2003)

${ }^{\odot 2003, ~ E d i t r i c e ~ K u r t i s ~}$

vestigation of intermittent iv administration of aminobisphosphonate, which showed an increase in BMD of the spine and femoral neck after 3 monthly infusions of pamidronate and ibandronate $(6,7)$. Ibandronate [1-hydroxy-3-(methylpentylamino)-propylidene bisphosphonates] is a potent aminobisphosphonate with proven efficacy in the treatment of malignant hypercalcemia (8) and of post-menopausal osteoporosis (9). For the treatment of osteoporosis, iv ibandronate, $2 \mathrm{mg}$ every 3 months as bolus injection, seems to be effective (7). The goal of the present open pilot study was to investigate the effect of $2 \mathrm{mg}$ of ibandronate, administered every 3 months over 2 yr, on BMD and on biochemical markers of bone metabolism in men with primary osteoporosis.

\section{MATERIALS AND METHODS \\ Study subjects}

The patients (age 40 to $80 \mathrm{yr}$ ) were referred to the out-patient clinic for osteoporosis at the University Hospital of Lausanne, Switzerland. This pilot study was open and monocentric. The inclusion criteria were the presence of one osteoporosis fracture (vertebra, femoral neck or wrist) and a BMD at the lumbar spine of at least $1 \mathrm{SD}$ below the Z-score, or at least $1.5 \mathrm{SD}$ below the Z-score without any fracture. Men with secondary causes of os- 
teoporosis were ineligible, as were those with other bone diseases, vitamin $D$ deficiency, renal disease (indicated by a serum creatinine concentration $>160 \mathrm{~mol} / \mathrm{l}$ ), known endocrinopathy affecting bone metabolism (hypogonadism, hyperparathyroidism, hyperthyroidism, etc.), medication affecting bone metabolism (corticosteroids or cyclosporine during the preceding 6 months; calcitonin, fluor or bisphosphonates during the preceding 12 months), a history of cancer other than basal-cell carcinoma of the skin, or a severe disability disease with a life expectancy of less than $2 \mathrm{yr}$. All patients had given their written informed consent prior to enrolment. The study was approved by the local Ethics Committee.

\section{Treatment}

All patients included in this study received $2-\mathrm{mg}$ ibandronate every 3 months as a bolus injection at $0,3,6,9,12,15,18$ and 21 months. All got oral supplementation with $1000-\mathrm{mg}$ calcium and $880-U I$ vitamin D every day during the $2 \mathrm{yr}$ of treatment.

\section{Baseline and follow-up study}

In the pre-treatment period, a complete history was obtained, and each patient underwent a physical examination, electrocardiography, chest radiography, and serum parameters [osteocalcin, C-telopeptides, PTH, $25(\mathrm{OH})$ vitamin D and 1,25 $(\mathrm{OH})$ vitamin $\mathrm{D}$, complete blood cell count, electrolytes, creatinine, albumine, liver-function tests]. The patients included returned for visits, biochemical measurements and treatment at $0,3,6,9,12,15,18,21$ and 24 months. The BMD of the lumbar spine (anteroposterior view), femoral neck, trochanter and distal forearm was measured by dual-energy X-ray absorptiometry (Hologic QDR 2000) at baseline and at 6, 12, 18 and 24 months. Quality control of the BMD measurements, including teaching of relevant technicians and phantom assessments, was performed before and throughout the study by checking accuracy and reproducibility on a daily basis. The precision of our Hologic QDR 2000 equipment was 1.3\% at the lumbar spine, $1.8 \%$ at the femoral neck and $1.9 \%$ at the trochanter (7). The precision was evaluated by repeated measurement in 10 subjects ( 4 measurements per subject) according to Gluer et al. (10). The long-term stability was monitored during the study by measuring an object of constant mass (European spine phantom) on daily basis. The device showed a high stability during all the study with an in vitro long-term reproducibility between 0.37 and $0.47 \%$ in BMD per year. All scans were reviewed by a blinded expert, and reanalyzed upon request. BMD is expressed in grams of hydroxyapatite per $\mathrm{cm}^{2}$. Posteroanterior and lateral radiographs of the lumbar and thoracic spine were obtained at base line and after 2 yr of treatment. All radiographs were reviewed by a blinded expert.

Fasting serum samples were obtained in the morning for the measurement of calcium corrected for albumine, phosphate, creatinine, alkaline phosphatase, osteocalcin, $\beta$-crosslaps, PTH. Plasma $25(\mathrm{OH})$ vitamin $D$, and 1,25 (OH) vitamin $D$ were measured on fasting serum sample at baseline and at 12 months. These parameters were determined by the following methods: osteocalcin by a two-site radioimmunoassay as described by Garnero et al. (11) (Elsa-Osteo, CIS bio international, Gif-surYvette, France; normal 9.6 to $26.8 \mathrm{ng} / \mathrm{ml}$ for men $>30 \mathrm{yr}$ ); serum $\beta$-crosslaps by a two-site radioimmunoassay (Systèmes Elecsys 1010/1020, Roche Diagnostics, Meylan, France); intact PTH with an immunoradiometric assay (Nichols Institute, San Juan Capistrano, California; intact PTH 100T Kit; normal 10 to $65 \mathrm{pg} / \mathrm{ml}$ ); $25(\mathrm{OH})$ vitamin $\mathrm{D}$ with a radioimmunoassay (DiaSorin, Stillwater, Minnesota, normal 8.4 to $52.3 \mu \mathrm{g} / \mathrm{l}) ; 1,25(\mathrm{OH})$ vitamin D with a radioimmunoassay (Incstar corporation, Stillwater, Minnesota). The correction of the serum calcium by albumine (normal 2.1 to $2.5 \mathrm{mmol} / \mathrm{l}$ ) was done according to statistics established by the laboratory of the hospital (12): measured calcium - 0.012 [measured albumine - 39.5 (g/l)]. Safety parameters measured included serum creatinine, sodium, potassium, alanine aminotransferase, aspartate aminotransferase, gamma-GT and complete blood count. All these latter determinations were done by routine laboratory methods.

\section{Statistical analysis}

The primary endpoint of the study was the lumbar spine BMD and the secondary endpoint was the effects on osteocalcin and $\beta$-crosslaps. The analysis of BMD variables, osteocalcin and $\beta$-crosslaps were based on the percent changes from baseline. Statistical differences were evaluated by paired t-test. $P$ values $<0.05$ were considered to be significant.

\section{RESULTS}

Fourteen consecutive male patients with primary osteoporosis were included in this study. Patient characteristics at baseline are given in Table 1. At entrance, 9 patients had experienced 18 vertebral fractures and 4 non-vertebral fractures (wrist 3; upper arm 1). All these fractures occurred, either spontaneously or after a minor trauma. Nine patients had a T-score below -2.5 DS either at the lumbar spine or femoral neck.

\section{$B M D$}

The BMD of the lumbar spine and of the trochanter increased significantly by $6.7 \pm 1.5 \%$ and by $3.2 \pm 0.8 \%$ (percent change, mean \pm SEM) after $2 \mathrm{yr}$ of treatment (Fig. 1). The BMD of the femoral neck and the total hip (Fig. 2) increased by $1.4 \pm 1.1 \%$ and $0.9 \pm 0.6 \%$, respectively. The BMD of the distal forearm increased by $1.5 \pm 0.5 \%$ (ns, data not shown).

\section{Biochemical markers}

As shown in Figure 3, there was a progressive decrease in bone serum $\beta$-crosslaps reaching a nadir between 9 and 12 months, and a slight increase

Table 1 - Patient characteristics at baseline (mean \pm SEM)

\begin{tabular}{ll}
\hline Age & $57 \pm 12 \mathrm{yr}$ (range: $40-73$ ) \\
BMI & $27 \pm 5 \mathrm{~kg} / \mathrm{m}^{2}$ \\
Fractures & $9 / 14$ patients \\
BMD lumbar spine & $-2.7 \pm 0.3 \mathrm{SD}$ (T-score) \\
BMD femoral neck & $-2.3 \pm 0.8 \mathrm{SD}$ (T-score) \\
\hline
\end{tabular}

BMI:body mass index; BMD: bone mineral density. 


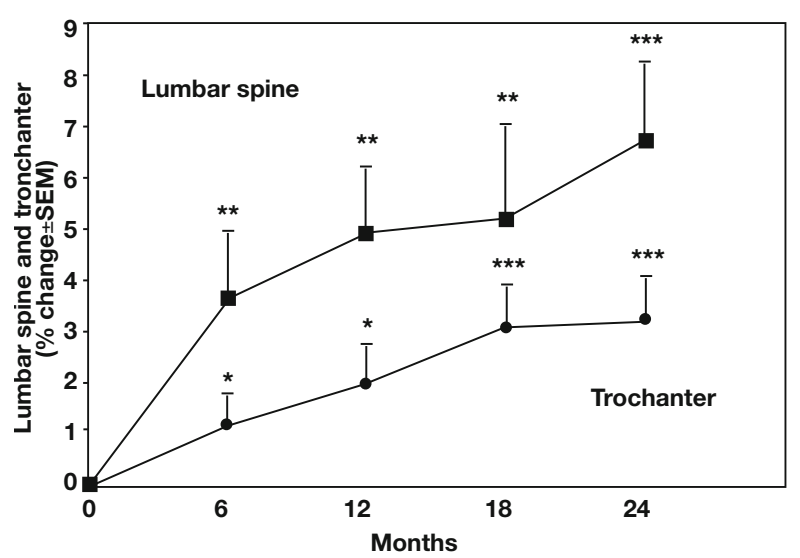

Fig. 1 - Mean ( \pm SEM) percent changes from baseline in the bone mineral density of the lumbar spine and the trochanter during $2 \mathrm{yr}$ of treatment with iv ibandronate. Asterisks indicate significant differences from baseline $\left({ }^{\star} p<0.05,{ }^{\star \star} p<0.01,{ }^{\star \star *} p<0.001\right)$.

during the second year of treatment at a level of $30 \%$ below the baseline. Osteocalcin decreased slower and remained stable at 30 to $40 \%$ below the baseline during the whole treatment. Corrected serum calcium increased slightly from $2.11 \pm 0.08$ to $2.24 \pm 0.09 \mathrm{mmol} / \mathrm{l}(\mathrm{p}<0.01)$ during the $2 \mathrm{yr}$ of treatment. PTH, $25(\mathrm{OH})$ vitamin D and 1,25 (OH) vitamin $D$ did not change during the 2 yr of the study.

\section{Fractures}

Four fractures occurred in four men: 2 new spontaneous vertebral fractures in 2 men with pre-existent vertebral fractures, and 2 traumatic fractures after a fall in 2 men (radius, metacarpus). The treatment was conservative in all cases.

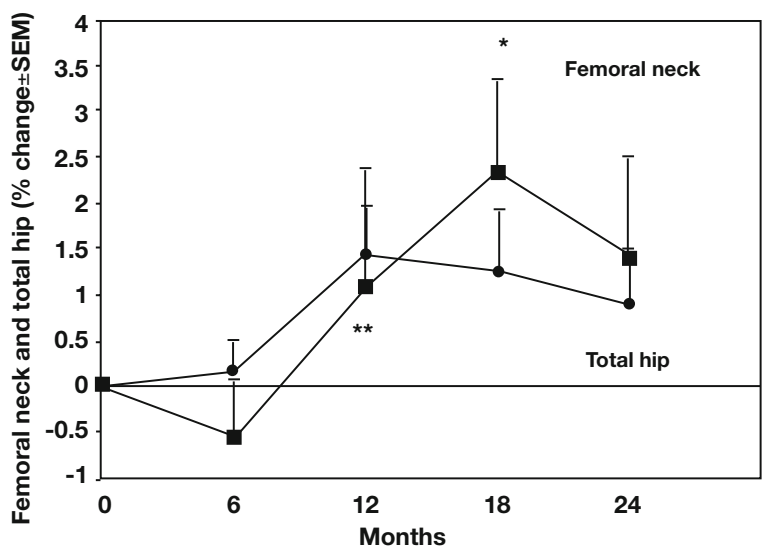

Fig. 2 - Mean ( \pm SEM) percent changes from baseline in the bone mineral density of the total hip and the femoral neck during $2 \mathrm{yr}$ of treatment with iv ibandronate. Asterisks indicate significant differences from baseline $\left({ }^{\star} p<0.05,{ }^{* *} p<0.01\right)$.

\section{Safety}

There were no serious drug-related adverse events and 38 non-serious adverse events during the study. One patient dropped out after $1 \mathrm{yr}$ and another after 18 months for reasons not related to ibandronate (one was depressed, one for unknown reason). Flu-like symptoms were reported in 3 patients: in 2 patients after the first and second injection and in 1 after each injection. Flu-like symptoms were controlled by self-administration of paracetamol for 1 or 2 days, and were not a cause of withdrawal. One patient experienced a transient skin rash 2 weeks after the last injection of ibandronate. The patient was examined by an allergologist, and no trigger was found. A transient relative eosinophilia occurred in one patient, and a transient relative monocytosis in another patient, without evident causes.

\section{DISCUSSION}

The patients included in this study had severe osteoporosis, considering the low BMD values and the prevalence of pre-existing fractures in two thirds of them (Table 1). Two-mg ibandronate given iv every 3 months for $2 \mathrm{yr}$ increased BMD at lumbar spine, trochanter and femoral neck. The magnitude of this effect was similar to that reported in men with osteoporosis after $2 \mathrm{yr}$ of treatment with $10-\mathrm{mg}$ oral alendronate given daily: 6.7 vs $7.1 \%$ at the lumbar spine, 3.2 vs $4.3 \%$ at the trochanter, and 1.4 vs $2.5 \%$ at the femoral neck (4). These increases in BMD are also similar to those reported in postmenopausal women with osteo-

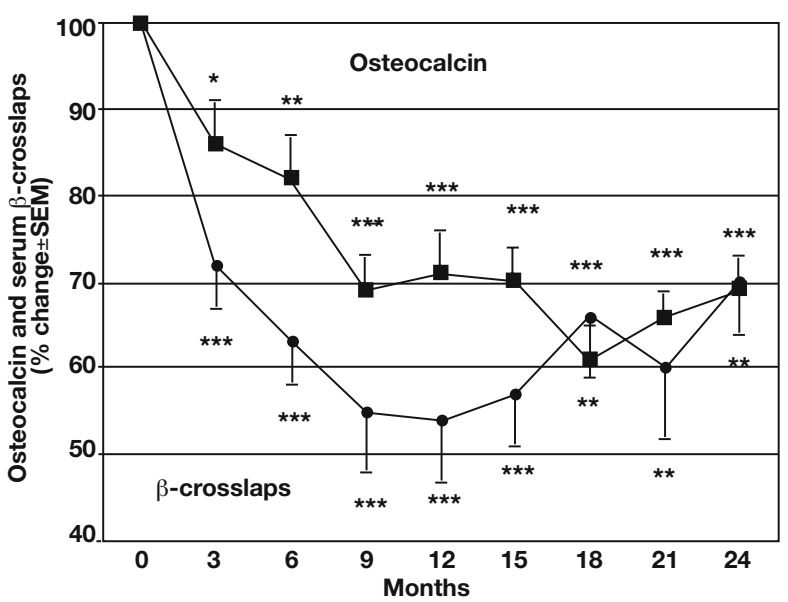

Fig. 3 - Mean ( \pm SEM) percent changes from baseline in serum osteocalcin and $\beta$-crosslaps during 2 yr of treatment with iv ibandronate. Asterisks indicate significant differences from base line $\left({ }^{*} p<0.05,{ }^{* *} p<0.01,{ }^{* *} p<0.001\right)$. 
porosis after $2 \mathrm{yr}$ of therapy with iv pamidronate or oral alendronate or risedronate $(6,13,14)$.

Serum $\beta$-crosslaps, a marker of bone resorption, decreased by 30 to $45 \%$, and osteocalcin, a marker of bone formation, by $30 \%$; these values were obtained 3 months after the last bolus of ibandronate, just before the next injection. The magnitude of the decline of the markers of bone remodelling was slightly less pronounced to that published with bisphosphonates in men or in women $(4,6,9,13,14)$. In these studies, osteocalcin decreased by 35 to $40 \%$, even by $50 \%(6,13)$. The comparison with markers of bone resorption is difficult. We used a serum marker, while the above mentioned studies used different urinary markers. The latter decreased by 26 to $40 \%$ in some studies $(6,14)$, and by 50 to $74 \%$ in other studies $(4,9,13)$. The pattern of the decline of the markers was slightly different to that described in other studies with bisphosphonates. The maximum decrease was reached only after 9 to 12 months, while it is usually obtained already after 3 to 6 months. However in women with osteoporosis, the injection of 2-mg ibandronate every 3 months decreased osteocalcin progressively with a nadir only after 9 months (7). In that study, urinary C-telopeptide decreased by nearly $70 \% 1$ month after the injection of 2-mg ibandronate, but increased again at nearly $50 \%$ at 2 months. This intermittent decline of the resorption markers raises the question if 3 months are an appropriate interval between two iv doses of ibandronate. A significant association between pharmacokinetics of oral ibandronate and clinical response in bone turnover and bone mass in women with post-menopausal osteoporosis was shown (15). A recent preliminary report confirms that, after $1 \mathrm{yr}$ of treatment, the increase of BMD and the suppression of bone markers achieved with 2-mg iv ibandronate given every 3 months in post-menopausal osteoporosis were similar to those achieved with oral bisphosphonates (16). These results suggest that 3 months are an appropriate interval between two iv doses of ibandronate, when $2 \mathrm{mg}$ are given.

The increase of the corrected serum calcium level probably reflects the positive effect of supplementation with calcium and vitamin D. An immediate and transient drop in serum calcium and rise in PTH have been invariably found with iv bisphosphonates. We could not observe such modifications, since the measurements were repeated only after 3 months, just before the next infusion of ibandronate.

Flu-like symptoms were the only side effect reported. They were well controlled with paracetamol, and were not a cause of withdrawal. Flu-like symptoms were described in approximately $20 \%$ of women with osteoporosis treated with either 20-mg iv ibandronate, or a perfusion of $30-\mathrm{mg}$ pamidronate $(6,7)$. Our study has several limitations: it is an uncontrolled study, and the number of included patients was small. In conclusion, this pilot study confirms that iv ibandronate is a promising treatment for osteoporosis in men. The increases of BMD and the suppression of bone markers achieved with 2 mg given every 3 months were similar to the results obtained with oral bisphosphonates. The convenience of a bolus injection every 3 months and the good tolerance make this treatment a promising alternative to oral bisphosphonates.

\section{ACKNOWLEDGMENTS}

Results of this study have been presented in part at the following congresses and published as abstracts: World Congress on Osteoporosis, Chicago, USA, 2000 [Osteoporosis Int 2000, 11 (Suppl. 2): 188 (P492)]; $1^{\text {st }}$ Joint Meeting of the International Bone and Mineral Society and the European Calcified Tissue Society, Madrid, Spain, 2001 [Bone 2001, 28: S238 (P615S)].

\section{REFERENCES}

1. Cooper C, Campion G, Melton LJ III. Hip fractures in the elderly: a word-wide projection. Osteoporos Int 1992, 2: 285-9.

2. O'Neill TW, Felsenberg D, Varlow J, Cooper C, Kanis JA, Silman AJ. The prevalence of vertebral deformity in European men and women: the European Vertebral Osteoporosis Study. J Bone Miner Res 1996, 11: 1010-8.

3. Eastell R, Boyle IT, Compston J, et al. Management of male osteoporosis: report of the UK Consensus Group. QJM 1998, 91: 71-92.

4. Orwoll E, Ettinger $M$, Weiss $S$, et al. Alendronate for the treatment of osteoporosis in men. N Engl J Med 2000, 343: 604-10.

5. De Groen PC, Lubbe DF, Hirsch LJ, Daifotis A, Stephenson W, Freedholm D. Esophagitis associated with the use of alendronate. N Engl J Med 1996, 335: 1016-21.

6. Thiebaud D, Burckhardt P, Melchior J, et al. Two years' effectiveness of intravenous pamidronate (APD) versus oral fluoride for osteoporosis occurring in the postmenopause. Osteoporos Int 1994, 4: 76-83.

7. Thiebaud D, Burckhardt $P$, Kriegbaum $H$, et al. Three monthly intravenous injections of ibandronate in the treatment of postmenopausal osteoporosis. Am J Med 1997, 103: 298-307.

8. Ralston SH, Thiebaud D, Herrmann Z, et al. Dose-response study of ibandronate in the treatment of cancer-associated hypercalcaemia. Br J Cancer 1997, 75: 295-300.

9. Riis BJ, Ise J, von Stein T, Bagger Y, Christiansen C. Ibandronate: a comparison of oral daily dosing versus intermittent dosing in postmenopausal osteoporosis. J Bone Miner Res 2001, 16: 1871-8.

10. Gluer CC, Blake G, Lu Y, Blunt BA, Jergas M, Genant HK. Accurate assessment of precision error: how to measure 
the reproducibility of bone densitometry techniques. Osteoporos Int 1995, 4: 262-70.

11. Garnero P, Grimaux M, Demiaux B, Delmas PD. Measurement of serum osteocalcin with a human specific two-site radioimmunoassay. J Bone Miner Res 1992, 7: 1389-97.

12. Kaufmann Didisheim N. Définition de l'intervalle de référence de la calcémie dans une population hospitalière. Doctor's thesis, Faculty of medicine, University of Lausanne, Switzerland, 1989.

13. Pols HAP, Felsenberg D, Hanley DA, et al. Multinational, placebo-controlled, randomised trial of the effects of alendronate on bone density and fracture risk in postmenopausal women with low bone mass: results of the FOSIT study. Osteoporos Int 1999, 9: 461-8.
14. Harris ST, Watts NB, Genant HK, et al. Effects of risedronate treatment on vertebral and nonvertebral fractures in women with postmenopausal osteoporosis: a randomised controlled trial. JAMA 1999, 282: 1344-52.

15. Ravn P, Neugebauer G, Christiansen C. Association between pharmacokinetics of oral ibandronate and clinical response in bone mass and bone turnover in women with postmenopausal osteoporosis. Bone 2002, 30: 320-4.

16. Adami S, Delmas P, Felsenberg D, et al. Three-monthly 2 mg intravenous ibandronate bolus injections significantly increase BMD in women with postmenopausal osteoporosis. IOF World Congress of Osteoporosis, Lisbon, Portugal, 2002, p S14-5 (abstract). 